\title{
Verwaltung zwischen Altruismus und institutionalisiertem Dilemma
}

\section{von Franz Thedieck und Bernd Banke}

\section{Einleitung}

Die Verwaltung tritt uns als altruistische Institution gegenüber: Sie dient in der Regel nicht sich selbst, sie ist kein „Selbstzweck“, sondern verfolgt regelmäßig andere, meist übergeordnete Ziele. Als der ausführende Arm der Politik soll sie deren politische Zwecke umsetzen, gleichzeitig aber, so zumindest in modernen, rechtsstaatlich organisierten Demokratien und unter dem Stichwort "public governance" Recht und Gesetz achten sowie Gemeinwohlinteressen wie Nachhaltigkeit und/oder soziale Gerechtigkeit durchsetzen. Alle diese Begriffe stehen für ethisch-moralische Werte, die, das ist schon auf den ersten Blick erkennbar, nicht immer oder nur mit Schwierigkeiten in Einklang mit gesetzlichen Vorschriften und politischen Vorgaben gebracht werden können. Die Menschen, die in dieser Verwaltung arbeiten, sind daher in besonderem Maße der Gefahr von Zielkonflikten ausgesetzt. Wie kann ich im Sozialamt zugleich gerecht, sparsam, legal und menschenfreundlich sein? Die öffentliche Verwaltung befindet sich durch ihre Einbindung in verschiedene Kontexte wie beispielsweise Politik und Recht permanent im Spannungsfeld unterschiedlicher Interessen. Dies trifft vor allem die in der Verwaltung tätigen Menschen. Dilemma-Situationen sind für sie praktisch institutionell angelegt. Versuche, ethische Erwägungen und moralische Werte einzubeziehen, verschärfen diese Problematik, obwohl feststeht, dass es der 
Einbeziehung dieser Werte in hochkomplexen Gesellschaften bedarf. Um den Betroffenen eine Hilfestellung zur Lösung solcher Dilemmata zu geben, schlagen die Autoren ein zweistufiges Verfahren vor. In einem ersten Schritt sollen Dilemmata mit Hilfe der Theorie Sozialer Systeme analysiert werden, um die strukturellen Kopplungen der Systeme, die die Dilemmata verursachen, bestimmen zu können. In einem zweiten Schritt wird die Tugendethik insbesondere in Form der klassischen Beamtentugenden als Orientierungspunkt zur Bewältigung der Dilemma-Situationen vorgeschlagen. Eine der Hauptaufgaben der Ethik als wissenschaftlicher Disziplin ist es, dem Menschen Entscheidungshilfen für Situationen zu geben, in denen jede denkbare Handlungsalternative Vor- und/oder Nachteile hat, die ohne Weiteres nicht gegeneinander abgewogen werden können. Diese Situation wird als "Dilemma“ bezeichnet (statt aller: Blume 2003). Die Sozialverwaltung soll dafür sorgen, dass bedürftige Bürger die Mittel für ein menschenwürdiges Leben erhalten. Andererseits ist sie beispielsweise der politischen Zielvorgabe unterworfen, Geld einzusparen. Es ist eine Aufgabe der Ethik, für solche Situationen Regeln und Grundsätze zur Verfügung zu stellen, die zu ethisch und moralisch gerechtfertigten Entscheidungen führen.

\section{Ethisch-moralische Dilemmata der Verwaltung}

Dilemma-Situationen treten immer dann auf, wenn sich die moralisch-ethische Bewertung einer Situation von der Bewertung unterscheidet, die durch korrekte Anwendung der gesetzlichen Grundlagen vorgeschrieben wird. Die Situation des Verwaltungsmitarbeiters kann in Form einer Grafik mit vier Quadranten dargestellt werden, in der rechtliche und ethische Aspekte einer Handlung in einem Graphen dargestellt werden, auf dessen vertikaler Achse die ethische Bewertung einer Situation als positiver oder negativer Wert dargestellt wird. Auf der horizontalen Achse erscheint die Frage nach Legalität oder Illegalität einer Handlung ebenfalls als positiver oder negativer „Legalitätswert“. 


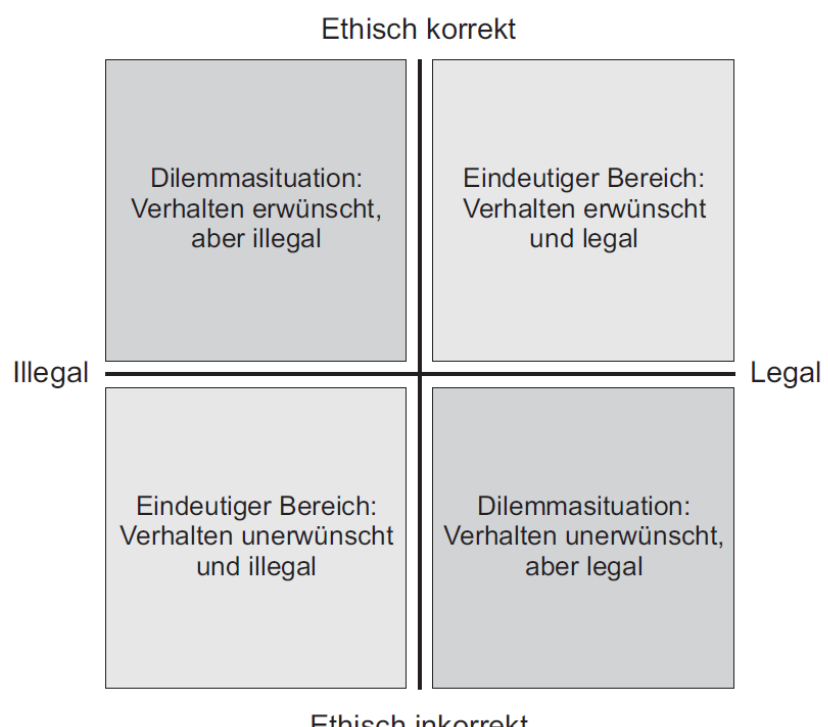

Abb. 1: Dilemma-Situationen in der Praxis

Eigene Darstellung; zur genauen Herleitung der Grafik vgl. Banke/Thedieck 2011, S. 461ff.

Der Frage, auf welchen Wegen Lösungsansätze für diese Dilemma-Situationen gefunden werden können, soll nun im Folgenden nachgegangen werden.

\section{Theorie sozialer Systeme - ein deskriptiver Ansatz}

Die in Deutschland traditionell vorherrschende intensive Rechtsstaatsdiskussion hat lange Zeit eine inhaltliche Auseinandersetzung zwischen Recht und Ethik verhindert, weil ein Gegensatz schwerlich denkbar war. Wenn das Recht am Gerechtigkeitsprinzip orientiert die Gesellschaft gestaltet, ist ein ethisches Dilemma schwer vorstellbar. Anders als sonstige Philosophien und Theorien zur Ethik und damit zu der die Menschen seit jeher beschäftigenden Frage: „Wie kann 
ich ein gutes und gelungenes Leben führen?" stellt der systemtheoretische Ansatz Niklas Luhmanns (1927-1997) nicht den Menschen selbst ins Zentrum der Untersuchung, sondern die kommunikativen Interaktionen innerhalb sozialer Systeme, genauer gesagt innerhalb des Systems Gesellschaft.

Nach den Forschungen Luhmanns besteht die moderne Gesellschaft aus verschiedenen Funktionssystemen. Jedes dieser Systeme übernimmt bestimmte Aufgaben, Funktionen, um Bedürfnisse einer Gesellschaft befriedigen zu können. Das Funktionssystem Politik dient in diesem Modell der Zuteilung und Ausübung von Macht. Das System Wirtschaft betreibt die Verteilung von Gütern und Dienstleistungen, das Funktionssystem Recht dient der Konfliktregulierung usw. (vgl. die Übersicht bei Krause 2004, S. 50). Nach der Theorie Luhmanns beobachten sämtliche dieser Teilsysteme einer Gesellschaft ihre Umwelt und bedienen sich dabei eines binären Codes mithilfe dessen sie ihre Beobachtung der Umwelt klassifizieren können. So werden Beobachtungen des Teilsystems Politik immer dadurch bezeichnet, dass sie mit dem Code „Amt/kein Amt“ oder etwas einfacher „gewählt/nicht gewählt“ beschrieben werden. Das Funktionssystem Recht, das in einer Gesellschaft die Funktion der Konfliktregulierung übernimmt, klassifiziert seine Beobachtungen nach dem Code „Recht/Unrecht“ oder „legal/illegal“, das System Wirtschaft nutzt den Code „Zahlung/keine Zahlung“ oder auch einfacher „Profit/kein Profit“ (ebd.).

Die Schwierigkeit dieser Arbeitsweise der einzelnen Funktionssysteme ergibt sich daraus, dass diese Codes nach den Feststellungen Luhmanns nicht kompatibel sind, das heißt, die einzelnen Funktionssysteme die Codes der anderen Systeme nicht verstehen. So kann das System Wirtschaft die Unterscheidung des Systems Recht „Recht/Unrecht“ weder erkennen, noch verstehen. Dasselbe gilt im umgekehrten Fall. Ethik und Moral, so jedenfalls der klassische Ansatz der Systemtheorie (vgl. Luhmann 2008, S. 259), stellen ihrerseits kein Funktionssystem der Gesellschaft 
dar, sondern eine Kommunikationsform, die sich, insofern ähnlich wie die gesellschaftlichen Funktionssysteme, eines binären Codes zur Beobachtung ihrer Umwelt bedient. Die Codierung der Moral ist „gut/schlecht“ oder aber, da moralische Urteile immer Werturteile über andere sind, „Achtung/Missachtung“. Anders als den gesellschaftlichen Funktionssystemen ist der Moral keine für eine Gesellschaft notwendige spezifische Funktion zugewiesen. Moralische Kommunikation erscheint vielmehr in allen Funktionssystemen, ohne selbst ein spezifisches Bedürfnis der Gesellschaft zu befriedigen. Sie pauschaliert nach der Auffassung Luhmanns Werturteile über Personen sowie Institutionen und wirkt daher streiterzeugend und ist gewaltnah (vgl. Luhmann 2008, S. 260; Baraldi et al. 1997, S. 119). Als Beispiel für diese Aussage kann etwa auf moralisch hochmotivierte religiöse oder politische Fanatiker verwiesen werden, die im Namen ihrer Moral auch vor der Tötung anderer nicht zurückschrecken. Für Luhmann stellt Moral, beziehungsweise die moralische Kommunikation eine „fieberhafte Reaktion“ der Gesellschaft dar, die letztlich, wie das Fieber für einen Menschen auch, für die Gesellschaft gefährlich ist (so etwa in einem Interview, zu finden unter anderem unter: www.youtube.com/watch?v=Yoxxx TMK020; zuletzt aufgesucht am 5.7.2013).

Ethik hingegen untersucht die Moral und soll bei der Feststellung helfen, ob eine Moral oder ein moralisches Urteil selbst „gut“ oder „schlecht“ ist. Eine Aufgabe, die Moral selbst nicht leisten kann, da sie sich immer per se als ,gut" betrachten muss, um über andere urteilen zu können. Der Ethik kommt in diesem Kontext die Aufgabe zu, die Moral zu überprüfen und vor ihr zu warnen. Sie ist die „Reflexionstheorie der Moral“ (Luhmann 2008, S. 267) und ermöglicht nach Luhmann einen friedlichen und konstruktiven Diskurs zu einem bestimmten gesellschaftlichen Phänomen. So wie sich nach der klassischen Theorie Luhmanns die Codes der einzelnen gesellschaftlichen Funktionssysteme mit der Folge unterscheiden, dass diese die Codes anderer Systeme nicht wahrnehmen und 
verstehen können, so ist es den einzelnen gesellschaftlichen Funktionssystemen auch nicht möglich, den binären Code der Moral „gut/schlecht“ zu verstehen. Das heißt, dass moralische Urteile über ein gesellschaftliches Phänomen wie beispielsweise einen Gebühren- oder Subventionsbescheid in den Systemen Politik oder Recht zunächst nicht wahrgenommen werden, da deren Codierung „Recht/Unrecht“ beziehungsweise „Amt/kein Amt“ lautet. So lange also eine moralische Bewertung die Position eines Politikers als einem Teilsystem des Funktionssystems Politik nicht beeinflusst, wird diese moralische oder rechtliche Wertung nicht wahrgenommen.

Im Anschluss an die Nuklearkatastrophe von Fukushima im März 2011 beschloss die deutsche Bundesregierung im Juni 2011 den Ausstieg aus der Nutzung der Kernenergie bis zum Jahre 2022. Dieser Beschluss warf eine vorherige Entscheidung vom Oktober 2010 um, wonach die Nutzung der Kernenergie bis ins Jahr 2024 verlängert wurde. Damit reagierte die Bundesregierung rasant auf wachsende Bürgerkritik, die eine Wiederwahl hätte gefährden können. Trotz Inkompatibilität ihrer Codes reagieren in bestimmten Situationen die verschiedenen sozialen Funktionssysteme der Gesellschaft aufeinander, so dass ein Vorgang, der sich in einem der Systeme ereignet, zu Änderung der Kommunikation in einem oder mehreren anderen Systemen führt. Welches sind hier die Wirkungsmechanismen? Da keines der sozialen Funktionssysteme nach Luhmann in der Lage ist, den Code eines anderen Systems zu verstehen, muss die Operation eines Systems zunächst von einem anderen System beobachtet werden. Systemintern kann die Beobachtung dann in dem beobachtenden System zu einer Irritation führen, die wieder ausschließlich systemintern verarbeitet wird. Falls die Irritation schließlich im beobachtenden System zu einer systeminternen Reaktion führt, wird dies in der Systemtheorie als strukturelle Kopplung bezeichnet (vgl. Baraldi et al. 1997, S. 186 mit zahlreichen weiteren Nachweisen in den Werken Luhmanns). 
Im obigen Beispiel um den Ausstieg aus der Kernenergie äußerten sich Politiker verschiedener Parteien erst, nachdem in den Medien, dem sozialen Funktionssystem mit der Aufgabe der Nachrichtenproduktion und -verteilung, moralische Entrüstung über offizielle Atompolitik geäußert wurde.

\subsection{Theorie sozialer Systeme in der Verwaltungspraxis}

Es soll nun die Frage gestellt werden, was die Theorie sozialer Systeme für den Bereich Verwaltung, speziell die Verwaltungsethik leisten kann. Diese Frage wird besonders dringlich, da dieser Ansatz Moral als eine gefährliche Form der Kommunikation betrachtet und der Ethik lediglich die Funktion einer Warnung vor Moral zuweist. Die Ansicht, die Gesellschaft sei in Funktionssysteme aufgeteilt, die sich gegenseitig nicht verstehen und die nicht in der Lage sind, moralische oder ethische Unterscheidungen zu erkennen oder gar zu verstehen, schließt damit im Prinzip Ethik und Werte aus den Handlungsoptionen der Menschen in den Systemen aus. Der Beitrag, den die Theorie sozialer Systeme zur Diskussion um Ethik in der Verwaltung leistet, kann demzufolge sicher nicht darin bestehen, einen Weg zu gutem, gerechtem und sozialem Leben zu zeigen. Ihre Leistung besteht vielmehr darin, eine Methode vorzuschlagen, wie gesellschaftliche Vorgänge analysiert und verstanden werden können. Dennoch macht diese Theorie mit der Figur der strukturellen Kopplung aber auch die wechselseitigen Bezüge und Abhängigkeiten zwischen den Systemen und auch die Einflüsse moralischer Kommunikation sichtbar. Sie eröffnet damit den in der Verwaltung Tätigen die

Möglichkeit, gegenseitige Einflüsse der Systeme frühzeitig zu erkennen und sich darauf einzustellen. Die Funktion der Theorie sozialer Systeme ist zunächst eine 
analytische. Dilemmasituation: Sie ist ein Erklärungsmodell für den Ablauf gesellschaftlicher Vorgänge. Die eingangs für die Verwaltung diagnostizierte „institutionalisierte Dilemma-Situation“ wird unter dem Blickwinkel dieser Theorie klar. Wegen ihrer Aufgaben wird Verwaltung immer zugleich in mindestens zwei Funktionssystemen tätig, die ihrerseits aber verschiedene Codes zur Beobachtung der Umwelt nutzen und demzufolge Phänomene unterschiedlich bewerten. Die Energiewirtschaft nimmt die Verlängerung der Laufzeiten für Kernkraftwerke als geringere Kosten wahr und damit als mehr "Zahlung“. Bedingt durch die Reaktion der Medien droht deswegen aber ein Rückgang der Wählerstimmen. Aus Sicht des Systems Politik bedeutet dieses Risiko „kein Amt“ mehr. Die Verwaltung wird nun in ihrer Verantwortung gegenüber den Angehörigen aller beteiligten Systeme zwischen die verschiedenen Interessen gedrängt. Es entsteht die bezeichnete Dilemma-Situation, da die Politik die Vergünstigung gerne aufheben würde, dies aber zunächst nicht den demokratisch erlassenen Gesetzen entsprechen würde.

Aus der Abbildung 2 wird ersichtlich, dass aus Sicht der Politik die erwünschten Ergebnisse, die einer Wiederwahl zuträglich wären, für die Verwaltung eine Dilemma-Situation verursachen. Die Kürzung der Laufzeiten ohne gesetzliche Grundlage wäre illegal; eine Analyse, die mit Hilfe des systemtheoretischen Ansatzes zwingend und logisch erscheint. 


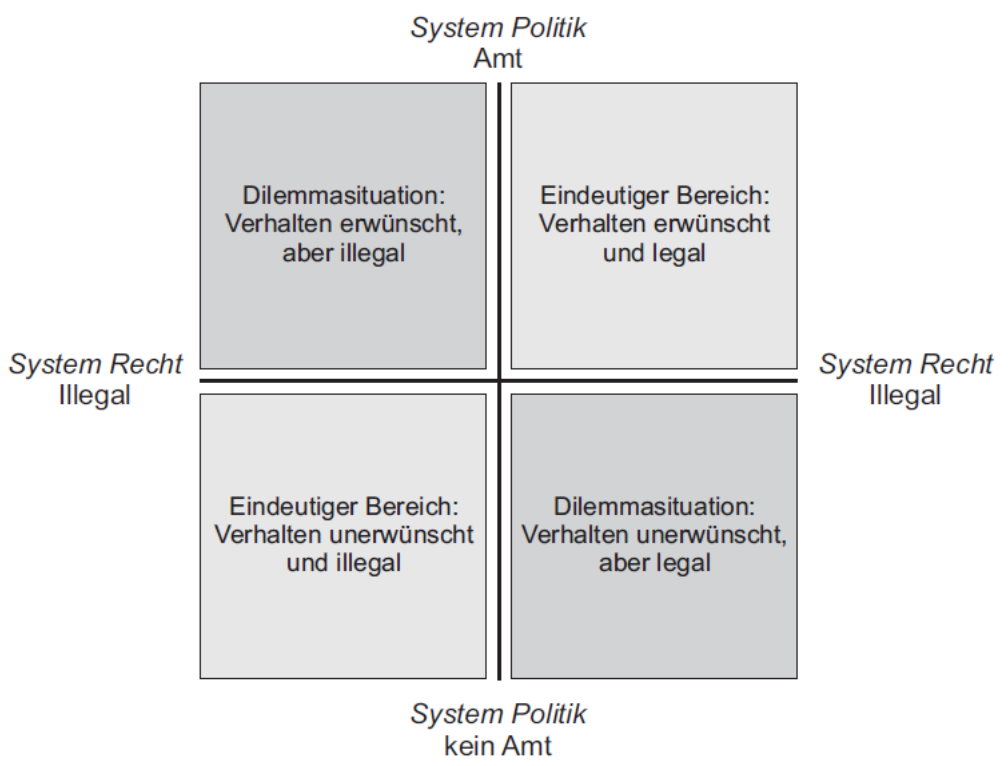

Abb. 2: Strukturelle Kopplung Politik/Recht

Eigene Darstellung

Gerade diese analytische Funktion und die eingangs beschriebene Tatsache, dass Verwaltungstätigkeit die Aufgaben anderer Funktionssysteme zu verwirklichen mindestens aber zu berücksichtigen - hat, macht die Theorie sozialer System für den Verwaltungsalltag besonders tauglich und hilfreich. Die Verwaltung ist über die Zuordnung $\mathrm{zu}$ den Systemen Recht und Politik hinaus in weitere soziale Funktionssysteme eingebunden. Sofern es sich um Verwaltung in Wissenschaft und Forschung handelt, wird sie sich mit dem Code und dem Kommunikationsmedium der Wissenschaft konfrontiert sehen. Die Wirtschaftsverwaltung muss sich dem binären Code „Zahlung/keine Zahlung“ der Wirtschaft stellen.

Dieser Ausgleich zwischen den Systemen macht es notwendig, dass ethischmoralische Werte in den durch strukturelle Kopplungen verursachten Dilemma-Situationen in die Entscheidungsfindung einbezogen werden. Eine Hilfe 
und einen Orientierungspunkt können materiale Werte liefern, wie sie in klassischen Ethiktheorien verkörpert sind. Hier fällt der Blick auf klassische Ansätze wie die Tugendethik.

\section{Materielle Werte der Verwaltung}

\subsection{Tugendethik}

Das Konzept der Tugendethik basiert auf Gedanken des griechischen Philosophen Aristoteles (384-322 v. Chr.). Aristoteles entwickelte die erste logisch-systematische Ethik, die auf dem Gedanken der Vernunft aufbaut. Über Thomas von Aquin (12251274) gewann Aristoteles in der frühen Neuzeit erheblichen Einfluss auf die christliche Philosophie und die wissenschaftliche Methodenlehre (Maas 2008, S. 45). Tomás Sedlácek bezeichnet Aristoteles als „vielleicht sogar“ den ersten exakten Wissenschaftler, der sich von einem Wissenschaftler der Moderne nicht unterscheidet (Sedlácek 2012, S. 154). Aristoteles hielt das Glück für das höchste Ziel des Menschen. Um dieses Ziel zu erreichen, bedarf es nach seiner Lehre einer vernünftigen und tugendhaften Ausrichtung des Lebens. Wie findet man aber die Tugenden, die dem Menschen helfen, sein höchstes Ziel zu erreichen?

Die Überlegung, dass Extreme generell nicht erstrebenswert sind, führt Aristoteles zu seiner Antwort. Nicht in der Maximierung liegt der Schlüssel zum Glück, sondern in der Ausgewogenheit der Mitte. Bei den menschlichen Eigenschaften sind zum Beispiel sowohl die Faulheit, als auch der Zustand des Workaholic negativ besetzt, sie stellen unerwünschte Verhaltensweisen dar. Deshalb muss die Tugend in der Mitte zwischen den Extremen gesucht werden. Das wäre im angegebenen Beispiel der Fleiß. Andere Extreme stellen die Tollkühnheit und die Feigheit dar, in der Mitte liegt die Tugend der Tapferkeit. In einem weiteren Beispiel bezeichnet man die Mitte zwischen Zügellosigkeit und Stumpfsinn als Selbstbeherrschung (vgl. 
Gfeller 1991, S. 69f.). Dass es nicht immer leicht ist, die Mitte zu erkennen und zu treffen, weiß auch Sedlácek: Auch deshalb ist es nicht leicht, ein tugendhaftes Leben zu führen (vgl. Sedlácek 2012, S. 162).

Das System der Wertegewinnung nach der aristotelischen Tugendethik lässt sich noch etwas komplexer am Bild eines Wertequadrats veranschaulichen:

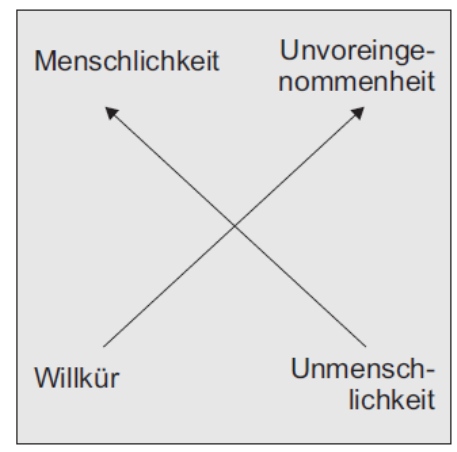

Abb. 3: Wertequadrat, Eigene Darstellung

Das Wertequadrat veranschaulicht die jeweils maßgeblichen Werte. Es ermöglicht dem Handelnden, sich im Geflecht widerstreitender Werte zu orientieren. Dies gilt natürlich auch für Mitarbeiter in der Verwaltung (mit dem Modell eines Wertequadrats arbeitet auch Wieland 2007, Abschnitt 4.1 für die Wirtschaftsethik). Doch zunächst soll die Tauglichkeit der Theorie an einem Beispiel aus dem Verwaltungsalltag getestet werden. Fall: Die nicht vollzogene Ausweisung Der Kurde Molla Karwen hat nach seiner Flucht nach Deutschland Asyl beantragt. In der Türkei hat er zuvor drei Jahre im Gefängnis verbracht, weil er den Wehrdienst verweigert hat. Gleichwohl wird sein Asylantrag letztinstanzlich abgelehnt und die Ausländerbehörde weist K. aus. Polizeioberkommissar Müller soll diesen Entscheid vollziehen und K. festnehmen und in die Abschiebehaft 
bringen. Müller kennt aus seinem bürgerlichen Engagement bei Amnesty International die Lebensbedingungen der Kurden in der Türkei und fürchtet, dass K. bei seinem Eintreffen dort menschenrechtswidrig behandelt wird. Daran möchte er sich nicht beteiligen und lehnt es ab, persönlich den Ausweisungsentscheid zu vollziehen. Einem Polizeibeamten kann der Staat nicht zubilligen, einer Weisung nach eigenem Gutdünken nachzukommen oder nicht. Das bedeutet aber nicht, dass er jede Entscheidung, die rechtmäßig zustande gekommen ist, zu vollziehen hätte. Vielmehr hat der demokratische Rechtsstaat in Deutschland aus den Menschenrechtsverletzungen während der Nazidiktatur die Lehre gezogen, dass der Verwalter sein Handeln an einer an allgemeingültigen Werten ausgerichteten Tugendethik überprüfen muss. In dem Beispielsfall sind diese Werte Menschenwürde, Unvoreingenommenheit und Aufrichtigkeit. Dem trägt § 36 Beamtenstatus $\mathrm{G}$ ausdrücklich Rechnung.

\subsection{Verantwortungsethik}

Der deutsche Soziologe Max Weber (1864-1920) lehnte die Tugendethik ab. Er bezweifelte, dass sittliche und moralische Werte die Wirklichkeit bestimmen und glaubte auch nicht an das Gute im Menschen (vgl. Prechtl 2008, S. 645). Andererseits ist das Versagen der Tugendethik vor den Herausforderungen der Realität nach Weber auch kein Grund, seine Augen vor der Wirklichkeit zu verschließen und sich auf eine Insel der Seligen zurückzuziehen. Vielmehr bleibt der Mensch für die Folgen seines Handelns verantwortlich, soweit er sie hätte voraussehen können. Er ist insoweit mitverantwortlich für die Gestaltung der Welt und die daraus erwachsenden Ergebnisse.

Man gewinnt fast den Eindruck, dass Weber in gewisser Weise die Verbrechen der NS-Herrschaft voraus geahnt habe. Auf jeden Fall hat er sie vorher bedacht. Aus 
dem sozialen Miteinander folgt nach Weber eine gemeinsame Verantwortung der Menschen für die Herstellung menschenwürdiger Lebensbedingungen. Eine Reduktion der Verantwortung auf den Bereich der eigenen Zuständigkeit, auf den subjektiven Anteil an dieser Welt wird sinnlos. Vor dem Unrecht und der Gewalt des Nationalsozialismus ist eine Exkulpation mit dem Hinweis, man habe auf Befehl gehandelt, ausgeschlossen. Deshalb haben solche Entschuldigungen vor Gericht auch keine Anerkennung gefunden und in die Beamtengesetze ist die Verantwortlichkeit der Beamten konsequent festgeschrieben worden. Zusammenfassend führt uns Max Weberzu der Einsicht, dass der Mensch sich nicht auf die Welt der Normen zurückziehen darf, sondern aus der Perspektive der gemeinsamen Mitwelt die Handlungsfolgen in seine Entscheidungen einbeziehen und mit verantworten muss. Der Organisationsexperte für Eisenbahnlogistik kann sich also nicht darauf zurückziehen, dass sein Tun wertneutral sei, weil den Organisationsentscheidungen an sich kein ethisches Unwerturteil anhaftet. Ihm ist vielmehr der Zweck der Transportbewegungen zuzurechnen, ja sogar für die willentliche Ausblendung dieses Elements der Organisationsentscheidungen wird der Entscheidungsträger explizit zur Rechenschaft gezogen. Deshalb durfte und konnte sich AdolfEichmann nicht auf die Neutralität seines Arbeitsgebiets verlassen. Ihm wie allen anderen Tätern des Unrechtsregimes wurden zu Recht die Folgen des verbrecherischen Gesamthandelns zugerechnet.

In unserem obigen Beispiel zur Verantwortung des Polizeibeamten löst die Verantwortungsethik ähnlich in dem Sinne den Ausweisungskonflikt, dass der Handelnde jederzeit prüfen darf und muss, ob sein Handeln im Einklang mit der Menschenwürde steht. 


\subsection{Die Beamtentugenden}

Versuchen wir nun im Anschluss an die Tugendethik die Werte oder Tugenden im Verwaltungssystem zu definieren, so können wir unschwer im Verwaltungshandeln abzulehnende Extreme wie Unmenschlichkeit und Willkür benennen. Der in der Mitte zwischen diesen Extremen liegende Wert kann als Unvoreingenommenheit beschrieben werden und kann zweifelsohne der Verwaltung als Richtschnur dienen. Liegt es da nicht nahe, in dieser Abwägung, der Suche nach der Mitte zwischen den Extremen, eine Parallele zum Grundsatz der Verbältnismäßigkeit zu sehen, der den Verwaltungsmitarbeiter zu einer ausgewogenen und angemessenen Entscheidung führen soll? Nach dem Verhältnismäßigkeitsprinzip dürfen Maßnahmen der Verwaltung keine Folgen auslösen, die erkennbar außer Verhältnis zu dem beabsichtigten Erfolg stehen. Obwohl er auch im einfachen Recht eine ausdrückliche Regelung gefunden hat (vgl. § 5 PolizeiG BW), folgt der Grundsatz direkt aus dem verfassungsrechtlichen Rechtsstaatsprinzip (vgl. ständige Rspr. seit BVerfGE 6, 389 [201f.]). Das würde bedeuten, dass einerseits die Aristotelische Tugendethik im modernen Rechtssystem noch immer eine prominente Rolle spielt und andererseits dass die Wurzeln des Verhältnismäßigkeitsprinzip bis in die klassische griechische Philosophie reichen.

Menschlichkeit und Unvoreingenommenheit stehen damit als erste Werte fest, die im öffentlichen Dienst Geltung beanspruchen können. Traditionell spricht man in diesem Zusammenhang auch von Beamtentugenden. Für alle Verwaltungen von Hochkulturländern sind Tugendkataloge aufgestellt worden: im frühgeschichtlichen Zweistromland, in China, Indien und Äthiopien (vgl. Röber/Huhnholz 2011, S. 115f.).

In Deutschland reichen die Anfänge der öffentlichen Verwaltung bis in das 15. Jahrhundert zurück; unser modernes Beamtenethos wurde stark von preußischen Traditionen beeinflusst. In seinen politischen Testamenten von 1752 (Friedrich der 
Große 1974) und 1769 legt der preußische König Friedrich II. (1712-1786) die Grundlage seiner Ethik klar. Sie könnte auch als Prinzip einer Ethik des öffentlichen Dienstes gelten; in der Einleitung des Testaments von 1769 formuliert er: „Unser Leben ist ein eiliger Übergang vom Augenblick unserer Geburt zu dem unseres Todes; während dieses kurzen Zwischenraumes ist der Mensch bestimmt, für das Wohl der Gesellschaft, an deren Körper er Glied ist, zu arbeiten.“ (Friedrich der Große 2011, S. 67)

Teile dieser Tradition haben sich bis in die Gegenwart erhalten: Die unparteiische Amtsführung z.B. in $\S 60$ Bundesbeamtengesetz (BBG), der volle persönliche Einsatz im Beruf in § 62 Abs. 1 BBG und die Loyalität in § 64 BBG (mit ähnlichen Ergebnissen Röber/Huhnholz 2011, S. 119). Zu den bedeutendsten Tugenden des preußischen Beamten zählen:

- Gehorsam und Loyalität,

- nimmermüde Wachsamkeit,

- ständiger Fleiß und

- unerschütterliche Ehrlichkeit (Hubatsch 1982, S. 173).

Dabei handelt es sich freilich nicht um außergewöhnliche Anforderungen, die nur in Preußen an einen Beamten gestellt wurden. Sie waren eher Bestandteil jeder Beamtentugend, allerdings ließ deren praktische Umsetzung vielerorts zu wünschen übrig. Selbst im traditionellen wie im modernen China gelten dieselben Werte, nämlich zuvorderst Loyalität und Gehorsam. „Wer beim Staat arbeitet, gehört einem Kreis an, in dem Leistung weniger zählt als Loyalität und Gehorsam.“ (Ackeret 2012*, S. 11) Max Weber beschreibt die preußische Verwaltung als regelgebunden und professionell. Hierzu gehören personelle und organisatorische Komponenten. Zu den personellen gehören: 
- hauptberufliche Amtsausübung,

- Entgeltlichkeit,

- Berechenbarkeit,

- professionelle Ausbildung und das Laufbahnprinzip

Organisatorische Merkmale der „rationalen“ Verwaltung sind:

- Arbeitsteilung auf der Basis geregelter Zuständigkeiten,

- Amtshierarchie,

- Dienst- und Fachaufsicht sowie Aktenmäßigkeit.

Die Beamtentätigkeit wird von persönlichen Beziehungen und politischen Opportunitäten gelöst, also verobjektiviert. Dies gilt sowohl für die Prinzipien, die für die Berufung in das Beamtenverhältnis gelten, als auch für die Grundsätze der Amtsausübung (vgl. Weber 1972, S. 124). Dieses idealtypische Bild einer Verwaltung verbindet sich leicht mit einer institutionenethischen Auffassung; aber es lassen sich auch einige individualethische Grundsätze für die Amtstätigkeit erkennen:

- eine Amtsausübung ohne persönliche Interessen,

- die Rechtfertigung des individuellen Verhaltens aus Recht und Gesetz und

- das Ausschließen jeglicher Form von Korruption.

Zusammenfassend bestehen die traditionellen Beamtentugenden aus folgenden Sekundärtugenden:

- Fleiß,

- Wachsamkeit und

- Pünktlichkeit. 
Daneben gelten aber auch traditionelle Primärtugenden:

- Menschlichkeit,

- Unvoreingenommenheit (Neutralität),

- Loyalität,

- Gehorsam,

- Ehrlichkeit und damit auch

- Unbestechlichkeit.

Hinzu kommen noch einige wenige moderne Tugenden:

- Verfassungstreue, die sich allerdings von der traditionellen Loyalität zum Herrscher ableitet, und

- selbstverantwortliches Einstehen des Beamten für sein eigenes Handeln.

Dieser Wert besitzt einen deutlichen Bezug zur Verantwortungsethik.

Diese Tugend der autonomen Verantwortlichkeit des Beamten macht nach den Erfahrungen des Dritten Reichs Schluss mit einer weit verbreiteten Entschuldigung für im Dienst begangenes Unrecht, nämlich dem Hinweis, man habe ja nur Befehlen von Vorgesetzten gehorcht. Eine solche Exkulpation ist heute nicht mehr zulässig (vgl. § 36 BeamtenstatusG, § 11 Abs. 1 SoldatenG).

\section{Schluss}

Die Schwäche der Luhmannschen Systemtheorie ergibt sich daraus, dass die einzelnen Funktionsbereiche nicht kompatibel sind und miteinander nicht kommunizieren. Sie stehen isoliert und beziehungslos nebeneinander und ermöglichen zwar eine Analyse eines einzelnen Funktionsbereichs, jedoch keine 
grenzüberschreitende Problemlösung. Die Systemtheorie liefert anerkanntermaßen brauchbare Ergebnisse innerhalb eines Systems, sie versagt aber notwendig, wenn Konflikte mehrere Systeme gleichzeitig betreffen.

Wer eine Konfliktlösung sucht, muss deshalb die Luhmannsche Theorie weiterentwickeln. Ein Weg führt über die „Strukturellen Kopplungen“. Diese sollen zeigen, dass die Systeme, die grundsätzlich die Codes der anderen Systeme nicht verstehen, dennoch in der Lage sind, die Vorgänge in einem anderen System zu beobachten und auf dieser Basis ihr Verhalten zu ändern. Lösungen werden allein dort möglich, wo diese Kopplungen erkannt und gesteuert werden können. Die Aussage, dass zwischen den Funktionssystemen einerseits keine Kommunikation möglich ist, andererseits dennoch eine „Strukturelle Kopplung“ stattfinden kann, ist nicht widerspruchsfrei. Deshalb wird hier ein anderer Lösungsweg vorgeschlagen, in dem Sinne, dass mit Hilfe der Tugendethik eine Entscheidung der Konflikte zwischen zwei verschiedenen Funktionsbereichen getroffen wird.

Die Verwaltung ist über die Zuordnung zu den Systemen Recht und Politik hinaus in weitere soziale Funktionssysteme eingebunden. Sofern es sich z.B. um Verwaltung in Wissenschaft und Forschung handelt, wird sie sich mit dem Code und dem Kommunikationsmedium der Wissenschaft konfrontiert sehen. Die Wirtschaftsverwaltung muss sich dem binären Code „Zahlung/keine Zahlung“ der Wirtschaft stellen. Dieser Ausgleich zwischen den Systemen macht es notwendig, dass ethischmoralische Werte in den Dilemma-Situationen in die Entscheidungsfindung einbezogen werden. Die entscheidende Orientierung liefern dabei die materialen Werte der Tugendethik. Der Mitarbeiter der Verwaltung darf sich in gravierenden Konfliktsituationen den Anforderungen der Tugendethik nicht entziehen, sondern muss sein Handeln an Hand ihrer Werte überprüfen. Im Ergebnis führt dieses Verfahren zu einem harmonischen Ausgleich zwischen der Systemtheorie und werteorientierten Ansätzen. 


\section{Quellen}

Ackeret (2012):

Aßländer M./Nutzinger H. G. (2010), Der systematische Ort der Moral ist die Ethik! Einige kritische Anmerkungen zur ökonomischen Ethik Karl Homanns. In: Zeitschrift für Wirtschafts- und Unternehmensethik (ZfWU), Jg. 11, S. 226-248

Banke, B. (2010): Studienbrief Ethik und nachhaltige Entwicklung. Kehl

Banke B./Thedieck, F. (2011): Verwaltungsethik. In: Rechnungswesen und Controlling in der öffentlichen Verwaltung, Gruppe 4, S. 455-477

Banke, B./Thedieck, F. (2012): Öffentliche Verwaltung ein institutionalisiertes ethisches Dilemma?! In: Verwaltung und Management, H. 5, S. 225-266

Baraldi, C./Corsi, G./Esposito, E. (1997): Glossar zu Niklas Luhmanns Theorie sozialer Systeme. Frankfurt/M.

Blume, T. (2003): Dilemma. In: Handwörterbuch Philosophie (hrsg. v. Wulff D. Rehfus). Göttingen, Oakville (Internet: www.philosophie-woerterbuch.de; zuletzt aufgesucht am 29.6.2013)

Faust, T. (2008): Verwaltungsethik in der Praxis „Harte“ und „weiche“ Gesichtspunkte. In: Zeitschrift für Wirtschafts- und Unternehmensethik, Jg. 9 (2008), H. 2, S. 244-262

Faust, T. (2012): Verwaltungsethik in Zeit und Raum. In: Verwaltungsrundschau, Jg. 5 (2012), S. 149-152

Friedrich der Große (1974): Das Politische Testament von 1752. Stuttgart

Friedrich der Große (2011): Das Politische Testament von 1769. In: Sander, U. C. (Hg.):

Friedrich der Große. Ausgewählte Schriften. Frankfurt/M.

Gfeller, N. (1991): Kleine Geschichte der Ethik. Zürich.

Hubatsch, W. (1982): Friedrich der Große und die preußische Verwaltung (2. Aufl.). Köln, Berlin

Krause D. (2004): Luhmann-Lexikon. Stuttgart

Lütge C. (2004): „Zum Vorteil aller“. In: FAZ vom 6.3.2004, S. 15 
Luhmann, N. (2008): 9. Paradigm Lost. In: Horster, D. (Hg.): Die Moral der Gesellschaft. Frankfurt/M., S. 259

Luhmann, N. (2002): Die Politik der Gesellschaft. Frankfurt/M.

Maas, J. F. (2008): Aristotelismus. In: Prechtl, P./Burkard, F. P.: Metzler Lexikon Philosophie (3. Aufl.). Stuttgart

Peltzer, U. (2011): Yang Jie und der westliche Astronaut. In Süddeutsche Zeitung vom 22.12.2011, S. 14

Prechtl, P. (2008): Verantwortungsethik. In: Prechtl, P./Burkard, F. P.: Metzler Lexikon Philosophie (3. Aufl.). Stuttgart

Röber, M./Huhnholz, K. (2011): Verwaltungsmodernisierung und Verwaltungsethik. In: Verwaltung und Management, 3, S. 115-133

Sommermann, K.-P. (1989): Brauchen wir eine Ethik des öffentlichen Dienstes? In: Verwaltungsarchiv, Bd. 89, S. 290-305

Sedlácek, T. (2012): Die Ökonomie von Gut und Böse. Berlin

Stober, Rolf (2011): Allgemeines Wirtschaftsverwaltungsrecht. Stuttgart

Weber, M (1972): Wirtschaft und Gesellschaft. Grundriss der verstehenden Soziologie (5. Aufl.; 1. Aufl. veröffentlicht 1921/1922). Tübingen

Wieland, J. (2007): Die Ethik der Governance (5. Aufl.). Marburg Internet Gabler Wirtschaftslexikon: http://wirtschaftslexikon.gabler.de/Definition/verwaltung.html Luhmann, N.: www.youtube.com/watch?v=YoxxxTMK020, zuletzt aufgesucht am 10.2.2012

\section{Erstveröffentlichung}

Lück-Schneider, Kraatz (Hg.)

Kompetenzen für ein zeitgemäßes Public Management, 2014

Prof. Dr. iur. Franz Th edieck († 2017), Kehl

Prof. Dr. iur. Bernd Banke, Reutlingen 